\title{
LA TERAPIA OCUPACIONAL EN EL MARCO DE LA SEGURIDAD SOCIAL EN SALUD ${ }^{1-2}$
}

El presente documento contiene algunas reflexiones acerca del quehacer profesional del Terapeuta Ocupacional, para tal fin se presentan las políticas en salud, la seguridad social en salud en Colombia y algunas implicaciones de la Terapia Ocupacional y la Seguridad Social en Salud.

El desarrollo profesional para las próximas décadas debe dirigirse hacia la formación profesional, la investigación, el líderazgo en la prestación de servicios de rehabilitación basados en la ocupación, el fortalecimiento gremial y el mercadeo social. Muy seguramente al trabajar sobre estas líneas de acción contribuiremos a fortalecer la identidad profesional, asumir la responsabilidad social que se tiene como profesionales ante la sociedad y el país y así mejorar la imagen pública y asegurar la viabilidad de la profesión.

\section{POLÍTICAS EN SALUD}

\section{Antecedentes}

A finales del siglo pasado en Alemania(1883-1889), Bismark plantea un modelo de seguro social que cubre solamente al trabajador de la industria. Dicho modelo basado en el concepto de riesgo, enfocado a trabajadores asalariados de bajos ingresos y cuya estructura financiera era la cotización soportada en el salario. Para el resto de la población los problemas de salud se solucionaban en forma caritativa ó eran responsabilidad de la familia y de las abuelitas. La tecnología era precaria y enfocada a la disminución del dolor.

\footnotetext{
1 GARCÍA RUIZ, Alix Solángel.Terapeuta Ocupacional. U.N. Magíster en Desarrollo social y educativo. U.PN. Especialista en Seguridad Social. U. Externado.

2 Conferencia presentada en el XI Congreso Nacional de Terapia Ocupacional. Noviembre de 1998.
} 
Como consecuencia de la segunda guerra mundial, la situación de salud y el alto número de personas con discapacidad, Beverige en Inglaterra y basado en la teoría económica Keynesiana, plantea un modelo de seguridad social para satisfacer las necesidades sociales de toda la población. Este modelo es en esencia solidario, proteccionista, financiado con impuestos y en busca de la equidad.

Hacia 1948, se crean la ONU y la OMS y uno de sus primeros aportes es definir la salud "La salud no solo es ausencia de enfermedad sino completo bienestar físico y mental".

En las décadas de los 50-60 el conceprto de salud gira en torno a la Enfermedad, hay proliferacion de instituciones Medicas, y se le asigna al Médico la reponsabilidad de la misma.

En los 70's, con los trabajos de Lalonde y Blum (Canadá) se integran por primera vez los conceptos ambientales, genéticos, conductuales y de servicios de salud que se enfoca hacia la promoción y prevención.

Alma Ata, 1978, plantea la Atencion Primaria basada en la promoción y prevención en contraste al concepto de salud de los años 60's basado en la enfermedad. Las políticas de salud se enfocan al fomento y la prevención como aproximaciones que tienen impacto en la calidad de vida.

En los 80 's, la política económica toma ventaja sobre la política social, especialmente por la deuda externa; en este proceso la salud pasa de las manos del médico, al salubrista y de este al economista. Por primera vez es considerada como un producto dentro del mercado. Al ser considerada como un producto, los economistas identifican la explosión de costos que se relaciona con el envejecimiento de la población, la utilización de tecnologías de punta y el aumento de la demanda de los servicios de salud.

En 1987, el Banco Mundial" en su informe "financiación de los servicios de salud", propone el cobro de aranceles a los usuarios de los servicios de salud, la provisión

3 BANCO MUNDIAL citado por RESTREPO, M., La Reforma a la Seguridad Social en Salud de Colombia. Preparado para la CEPAL. Documento multicopiado. Santa Fe de Bogotá. 1996. 
de mecanismos de seguro de salud y el empleo eficiente de los recursos no gubernamentales para la salud a través de instituciones públicas, privadas y la descentralizacion de los servicios de salud

En los 90's toman auge doctrinas económicas como el neoliberalismo que tiene expresiones muy concretas en la reorientación del gasto público, la apertura, la privatización y la descentralización, características que intervienen en todos los sectores sociales entre ellos salud.

En 1993, el Banco Mundial", en el informe "invertir en salud", propone como principios: los estados deben promover que las familias mejoren sus condiciones de salud, se reoriente el gasto público en programas que ayuden a los pobres y exista diversidad en el financiamiento y en la prestación de los servicios, muy de la mano con los planteamientos anteriores.

Las posteriores reformas combinar los sistemas públicos con las ventajas de los sistemas privados. Se dan entonces dos tendencias conceptuales que se han definido para guiar la asignación de recursos y la organización de los servicios dentro del sector salud, ellas son: regimen de la oferta y regimen de la demanda que implican desiciones políticas guíadas por teorías económicas.

\section{ENFOQUES DE LA ATENCIÓN EN SALUD}

\begin{tabular}{|c|c|}
\hline RÉGIMEN DE OFERTA & RÉGIMEN DE LA DEMANDA \\
\hline $\begin{array}{l}\text { - Los servicios de salud tienen características es- } \\
\text { peciales diferentes a otros bienes económicos. } \\
\text { - Enfasis en el papel del Estado } \\
\text { - El mecanismo es la ley, quien ordena que se debe } \\
\text { - Es centralista } \\
\text { - Se financia con impuestos } \\
\text { - Existe obligatoriedad del seguro } \\
\text { - Existe remuneración a los proveedores. } \\
\text { - Hay burocracia. }\end{array}$ & $\begin{array}{l}\text { - Los servicios de salud tienen las mismas caracte- } \\
\text { rísticas de cualquier bien económico. } \\
\text { - Énfasis en el papel del mercado. } \\
\text { - El mecanismo es la competencia (sube calidad y } \\
\text { baje precios). } \\
\text { - Se da regulación con marcos en los cuales puede } \\
\text { moverse. } \\
\text { - Es descentralizada. } \\
\text { - Los consumidores deciden si pagan un seguro de } \\
\text { - Elud o si pagan un servicio directamente. } \\
\text { - Genera de tarifas al proveedor es por servicios. } \\
\text { - Genos. }\end{array}$ \\
\hline
\end{tabular}

4 lbid. 
Las fallas del regimen de la demanda son (1) el aseguramiento, rompe con la equidad por los procesos de selección adversa ${ }^{5}$, selección de riesgo ${ }^{6}$ y los monopolios de las aseguradoras. (2) En la provisión de servicios, la naturaleza monopólica de los servicios hospitalarios, el poder monopólico de los profesionales (ej, delimitación de competencias entre los TF y TO) y asimetría de información entre proveedores y usuarios.

Las fallas del regimen de la oferta son el dominio de grupos diferentes al usuario, la corrupción, el clientelismo, la falta de de incentivos para innovar y proponer eficiencia, las desiciones basadas en ideología o intereses particulares y falta de sensibilidad a las necesidades del usuario.

Ya que ninguna de estas tendencias satisface las soluciones que se buscan a los problemas de salud. Aparece la teoría de la competencia regulada que persigue solucionar las fallas antes enunciadas.

\section{Teoría de la Competencia Regulada}

La competencia regulada es una estrategia de compra de servicios para obtener el máximo valor por el dinero que pagan los consumidores. Utiliza reglas que gratifican con más clientes $e$ ingresos a aquellos planes de salud que hacen un buen trabajo mejorando la calidad, disminuyendo el costo y satisfaciendo a los usuarios. El juicio del "buen trabajo", lo hace el patrocinador, armado con información adecuado y con el consejo de expertos y de usuarios bien informados y conscientes del costo de los servicios (Enthoven, 1993 citado por Restrepo 1996)

Los elementos más importantes de ésta teoría son: los protagonistas -el usuario, el patrocinador y el proveedor - y el flujo de información de calidad y de costos.

5 Selección adversa: cuando la persona se asegura porque está enferma.

- Selección de riesgo: la aseguradora toma medidas de manera que solo se puedan afiliar personas con poco riesgo de enfermarse. 


\section{Patrocinador}

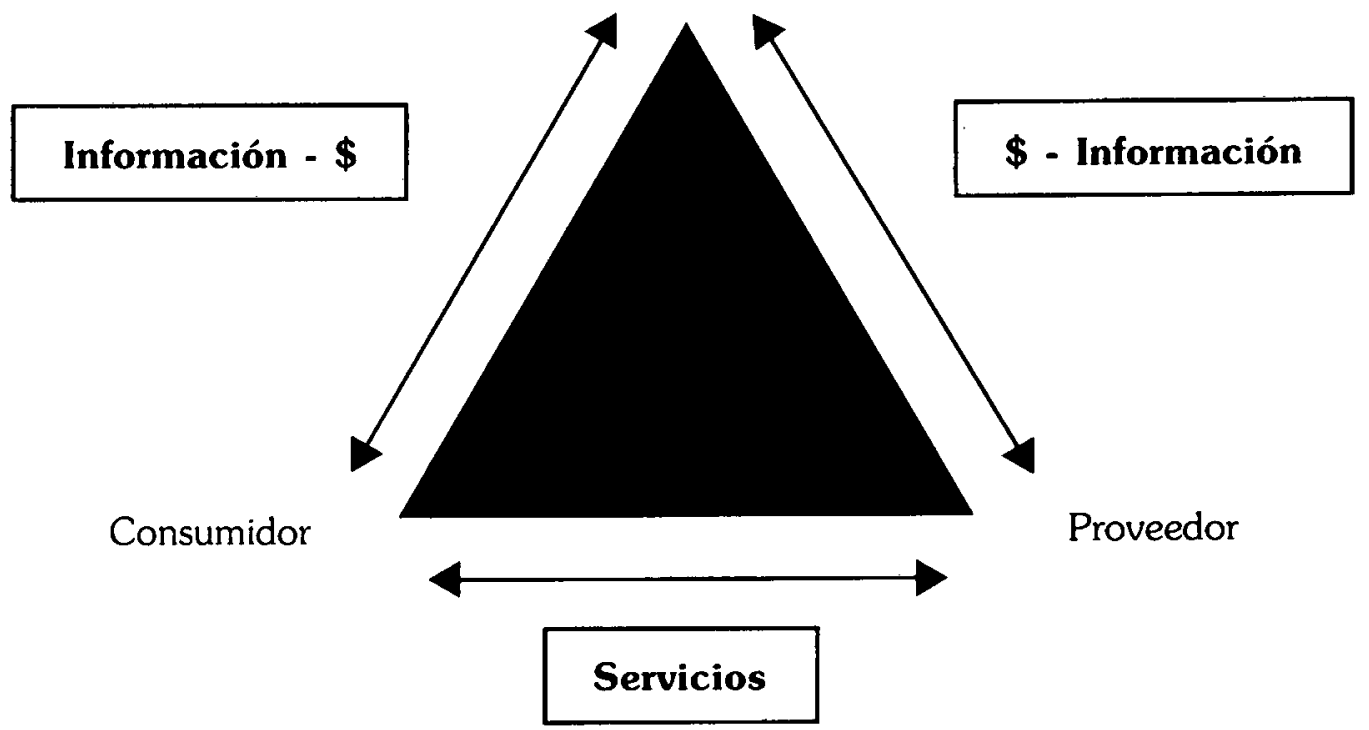

El patrocinador es una agencia que actúa como corredor y contrata un plan de salud, establece las reglas de equidad, administra el proceso de afiliación y maneja la selección de riesgo. En general lo que lo debe motivar es conseguir la atención en salud de la más alta calidad posible para sus beneficiarios. El proveedor son los profesionales de la salud de manera individual u organizados en instituciones quienes se convierten en unidades económicas que compiten entre sí y a través de las fuerzas del mercado desarrollan sistemas eficientes de prestación de servicios. El usuario o el consumidor es la persona o grupo familiar beneficiario de los servicios que presta el proveedor a través del patrocinador.

La relación entre los anteriores actores cambia con la época y con el modelo de salud que se maneje. La relación tradicional, cuando participa el usuario y el proveedor; el usuario paga al proveedor por el servicio y corre con el riesgo total de su salud. En la relación moderna aparece un asegurador donde el usuario paga al asegurador y este al proveedor por el servicio que preste al usuario; el riesgo lo asume el asegurador. La tendencia es la relación posmoderna, en la que entre el asegurador y el proveedor hay una relación contractual y el riesgo lo asume el proveedor. 


\section{LA SEGURIDAD SOCIAL EN COLOMBIA}

En Colombia, con la constitución de 1991 se realizan reformas estructurales en todos los sectores, entre ellos la salud. Por primera vez en Colombia se habla de la Seguridad Social ${ }^{7}$ en la que además de atender contingencias derivadas del proceso salud enfermedad, atiende en mayor o menor grado otras contingencias como son accidentes de trabajo y enfermedad profesional, maternidad, vejez, desocupación, invalidez y muerte. A través de riesgos profesionales, prestaciones económicas (pensiones y cesantías), salud y servicios sociales complementarios.

La salud en Colombia, era un sistema fragmentado: la salud privada, el seguro social y la salud pública, cada uno con debilidades y fortalezas. Fortalezas como la calidad y oportunidad de la salud privada, la tecnología y solidaridad del seguro social y la extensión geográfica y el modelo conceptual de la salud púplica, y como características generales el libre mercado de la salud privada, el monopolio del segruo social y la relación caritativa de la salud pública.

El modelo actual de salud, aparece con la constitución de 1991, la ley 10 de 1990, la ley 100 de 1993 y la ley 60 de 1993, los decretos reglamentarios, las resoluciones del Ministerio de Salud y los acuerdos del Consejo Nacional de Seguridad Social en Salud.

En la estructura del Sistema General de Seguridad Social en Salud se delimita la presencia del patrocinador representado en la Empresa Promotora de Salud (EPS), la Adminstradora de Regimen Susidiado (ARS) o el Estado, según sea el régimen (contributivo, subsidiado o vinculado) y además de las funciones enumeradas anteriormente está la de dar cuenta sobre el estado de salud de las personas, la promoción de la salud y poseer sistemas confiables de información.

El proveedor son los profesionales independientes o las instiuciones todos ellos llamados Instituciones Prestadoras de Servicios de Salud (IPS) ó las Empresas Socia-

7 Según José Manual Almanza, la Seguridad Social es el instrumento estatal, específico, protector de las necesidades sociales individuales y colectivas, a cuya protección preventiva, reparadora y recuperadora, tienen derecho los individuos, en la extensión, límites y condiciones que las normas dispongan, según permite su organización financiera. 
les del Estado (ESE), que prestan el Plan de Atención Básica (PAB) o el Plan Obligatorio de Salud (POS). Quienes deben poseer sistemas de garantía de la calidad y sustentar la práctica en guías de atención integral. Y el consumidor o los usuarios organizados a través de alianzas de usuarios.

Además cohexisten los entes de vigilancia y control y de financiación: Ministerio de Salud, Superintendencia Nacional de Salud y Fondo de Solidaridad y Garantía. Los elementos de regulación del mercado son la Unidad de Pago por Capitación (UPC), el Plan Obligatorio de Salud (POS), el Plan de Atención Básica (PAB), el porcentaje de cotización y las tarifas. La libre competencia entre el patrocinador o el asegurador (EPS) es por la calidad y entre proveedores (IPS) es por el precio.

Las fuentes de financiación provienen de los ingresos corrientes de la nación, los ingresos generales a nivel local, las contribuciones oligatorias, las cotizaciones de los usuarios y las tarifas o copagos de los usuarios.

\section{LA TERAPIA OCUPACIONAL Y LA SEGURIDAD SOCIAL EN COLOMBIA}

Existe una circunstancia común que motivó tanto la propuesta de Bismark, la de Beverige y por supuesto la de Colombia y fue la presencia de la discapacidad. En un principio como consecuencia de la guerra y más adelante como consecuencia del mundo moderno y esa misma circunstancia fue la que motivo en otras épocas al nacimiento de la Terapia Ocupacional.

La Terapia Ocupacional como profesión de la salud orienta su praxis al fomento de estilos de vida saludables, a la promoción del hombre saludable, evolutivo, productivo, lúdico, expresivo, creativo; a la prevención: detección precoz de deficiencias, discapacidades y minusvalías, y a la atención directa de personas con algún grado de discapacidad. En este sentido su actuar está en todos los planes de beneficios propuestos por el Sistema General de Seguridad Social en Salud: El Plan de Atención Básica (PAB) que camina en territorios de la promoción especialmente con algunos componentes de la prevención y el Plan Oligatorio de Salud (POS) que tiene acciones de prevención y en especial de atención directa o de diagnóstico, tratamiento y rehabilitación. 
La Terapia Ocupacional como la mayoría de profesiones de la salud en Colombia se encuentra en un momento de crisis social. Según Peñas en $1997^{8}$ y Cuervo y $\mathrm{col}^{9}$ en 1998 hay carencia de tecnología de avanzada, el uso de instrumentos formales es generalizado, existe inconsistencia en el uso de sistemas de clasificación diagnóstica, hay variabilidad en la duración de la sesión y tiempo de tratamiento Así mismo, la poca divulgación, la escases de investigación y el desconocimiento del código de ética. Esto sumado a que la situación actual de los profesionales de la salud en Colombia no es la mejor, teniendo en cuenta el desempleo, el subempleo, los salarios bajos y los sistemas de contratación (a término fijo y no idefinido) o por adscripción y la carencia de prestaciones sociales ${ }^{10}$. En este mismo orden de ideas, no hay demanda de Terapeutas Ocupacionales en entidades de vigilancia y control o hacedoras de políticas.

De otra parte, para Peñas en 1997, el Terapeuta Ocupacional posee autonomía en el proceso de selección de usuarios, en el establecimiento de objetivos y en la selección de estrategías. En cuanto al respaldo teórico son muchas las ganacias obtenidas en los últimos años, puesto que ello ha sido preocupación permanente de los Terapeutas, sinembargo a este respecto son muchos los caminos por construir. Para este fortalecimiento existen variedad de programas de postgrados interdisciplinarios en Colombia y en el exterior, espacios de agremiación generados por la Asociación Colombiana y espacios de participación social creados por la constitución y por la ley que deben ser apropiados por el terapueta ocupacional.

Por lo anterior, el desarrollo profesional para las próximas décadas debe dirigirse hacia la formación profesional, la investigación, el líderazgo en la prestación de servicios de rehabilitación basados en la ocupación, el fortalecimiento gremial y el mercadeo social. Muy seguramente al trabajar sobre estas líneas de acción contribuiremos a fortalecer la identidad profesional, asumir la responsabilidad social que

8 PEÑAS, Olga Luz. Autonomía profesional del Terapeuta Ocupacional. Tesis de Grado. Universidad Nacional. Facultad de Medicina. Departamento de Terapias. Unidad de la Ocupación Humana. Santa fe de Bogotá. 1998

9 CUERVO, C., ESCOBAR DE V., M. y TRUJILLO, A. Factores que determinan el impacto de los servicios de rehabilitación en las instituciones adscritas a la Secretaría Distrital de Salud. Universidad Nacional de Colombia y Secretaría Distrital de Salud. Santa Fe de Bogotá, 1998.

10 EL ESPECTADOR. La Federación tiene la palabra - Situación actual de los odontólogos. Santa Fe de Bogotá, 3 de octubre de 1998. 
se tiene como profesionales ante la sociedad y el país y así mejorar la imagen pública y asegurar la viabilidad de la profesión.

La formación profesional, es decir, abrirse a una formación contextual, dando interpretación a la razón de ser de la Terapia Ocupacional desde lo político, lo económico, lo social, lo jurídico y lo humano, en este mismo sentido, abrirse a lo interdisciplinario para fortalecer lo disciplinario.

Profundizar en lo técnico y en lo tecnológico, en el uso de instrumentos validados, unificar sistemas de clasificación diagnóstica, sustentar la práctica en guías basadas en la evidencia, promover el uso de tecnología de avanzada, fortalecer la autonomía en los procesos de selección de usuarios, establecimientos de objetivos y selección de estrategías. Apoyarse en mecanismos de calidad, auditoría y satisfacción de los usuarios.

La investigación, es necesario mostrar resultados a través de investigaciones, describir los servicios y proponer estándares de calidad. Esto debe ser medible para las necesidades de los administradores y financieros, pero no son clínicamente importantes o relevantes para los usuarios.

El líderazgo en la prestación de servicios de rehabilitación basados en la ocupación, implica gerenciar los servicios de Terapia Ocupacional: definir indicadores de impacto (cobertura, morbilidad...), de gestión (rendimiento, productividad, costos...), demostrar el costo-beneficio de los servicios de rehabilitación en años de vida saludable, en la disminuación de costos por hospitalización, etc. Dar cuenta a los aseguradores, gerentes y a la sociedad en general de la eficiencia, calidad y efectividad de los servicios.

Ser líderes también es mantener un comportamiento ético, que se manifiesta en la forma de actuar y decidir las cosas dentro del sentido de responsabilidad y absoluta sujeción a la moralidad y a las leyes, en la realización de actos ajustados a las normas de estricta consiencia, respeto a la dignidad de los seres y al reconocimiento de sus derechos, en la canalización de todas las acciones para el bien individual y colectivo. 
El fortalecimiento gremial, significa ser actor en la asociación, compartir con ella los logros y dificultades del quehacer profesional; debatir, confrontar y disernir juntos para construir el futuro de la Terapia Ocupacional, legitimando conductas y reconstruyendo el perfil profesional. Implica también utilizar los espacios de participación social para dar a conocer las ideas e intereses profesionales y reformular la ley de Terapia Ocupacional.

El Mercadeo Social, implica conocer la oferta y la demanda de la terapia ocupacional. Realizar las proyecciones epidemiológicas y demográficas para definir cuales son las careacterísticas de los posibles usuarios y del terapeuta ocupacional del mañana.

La idea es ofertar servicios agradables, confortables, accesibles, enmarcados en los planes de beneficios y que respondan a los juegos de la oferta y la demanda. Educar a los educadores, legisladores, aseguradores y otros profesionales sobre el arte de la Terapia Ocupacional compartiendo con ellos y con la sociedad en general lo que se hace que no hacen otros profesionales.

Por lo anterior cada terapeuta ocupacional debe asumir su responsabilidad profesional manteniéndose actualizado, dando diferentes significados a su quehacer, escribiendo y socializando sus logros, alimentando lo disciplinario de lo interdisciplinario, siendo miembros activos de la asociación, apoyando actividades para mejorar los servicios, de esa manera se estrá dando respuesta a la sociedad que confia en su actuar, para mejorar la imagen pública y asegurar la viabilidad de la profesión.

En este orden de ideas, se hace necesario conocer las características poblacionales del país que en la actualidad sugieren de un perfil profesional encaminado a programas de promoción de la salud y prevención de la enfermedad, el reconocimiento de estilos de vida saludables y la relación entre ocupación, salud y vida de los usuarios.

Asumir estos desafíos permitirán fortaleceer la identidad profesional, asumir la responsabilidad social que se tiene como profesionales ante la sociedad y el país y mejorar la imagen pública. 


\section{AGRADECIMIENTOS}

Es mi deseo agradecer a la Terapeuta Ocupacional Silvia Duarte y por intermedio de ella a la Junta Directiva de la Asociación Colombiana de Terapia Ocupacional por haberme invitado y motivado a escribir este documento. De igual manera, deseo agradecer a la Terapeuta Ocupacional Carmen Aleyda Fernandez M. por sus comentarios y reflexiones que alimentaron estas páginas y las ideas y comentarios del Enfermero Carlos Andrés Rincón C.

\section{REFERENCIAS BIBLIOGRÁFICAS}

BONTJE, Peter. Trends in Occupational Therapy - a worldwide perspective. Bulletin. WFOT. Vol 37. Japón. May 1998.

CARNEY, Terry and HANKS, Peter. Social Security in Australia, Ed. Oxford. First Edition, Melbourne, 1994

CUERVO, C., ESCOBAR DE V., M. y TRUJILLO, A. Factores que determinan el impacto de los servicios de rehabilitación en las instituciones adscritas a la Secretaría Distrital de Salud. Universidad Nacional de Colombia y Secretaría Distrital de Salud. Santa Fe de Bogotá, 1998.

EL ESPECTADOR. Seguridad Social en Salud - Análisis de la ley 100 de 1993. Santa Fe de Bogotá, 26 de septiembre de 1998.

EL ESPECTADOR. La Federación tiene la palabra - Situación actual de los odontólogos. Santa Fe de Bogotá, 3 de octubre de 1998.

HOPKINS \& SMITH. Terapia Ocupacional. Willard / Spackman. Editorial Panamericana. Octava Edición. España. 1998.

JONES, DIANNE, MILLER, Advocacy for community health. Community Health Policy and practice in Australia. Edited by Pluto Press Australia. First Edition. 1992.

KOTLERTO PHILIP, Roberto, Eduardo. Mercadotecnia Social - Estrategias para cambiar el comportamiento público. Editorial Diana. México. 1993.

MINISTERIO DE SALUD. Ley 100 de 1993. La Seguridad Social en Colombia. 1994. 
PEÑAS, Olga Luz. Autonomía profesional del Terapeuta Ocupacional. Tesis de Grado. Universidad Nacional. Facultad de Medicina. Departamento de Terapias. Unidad de la Ocupación Humana. Santa fe de Bogotá. 1998

RESTREPO TRUJILLO, Mauricio. La reforma a la seguridad social en salud de Colombia y la teoría de la competencia regulada. Preparado para la CEPAL. Documento multicopiado. Santafé de Bogotá. 1996

RUEDA, C. y PINZÓN, C. Uso de indicadores y herramientas fundamentales para la administración de servicios de salud. Revista Vía Salud. No. 3 . Santa Fe de Bogotá, 1998.

TRULLIJO, Alicia. Reflexiones sobre la identidad del terapeuta ocupacional. Revista acción Vol2 No. 1. Santa Fe de Bogotá. Julio de 1998.

TRUJILLO, Alicia. Código de procedimientos de evaluación y tratamiento de terapia ocupacional: una propuesta. Revista Acción Vol. 8 No. 1. Santa Fe de Bogotá. 1998.

VIANA DA CRUZ, T. Clarificando ideas para ver posibilidades. Revista Materia Prima. Año 3 No. 8. Buenos Aires. Argentina. 1998. 